\title{
Nuclear lamins and diabetes mellitus
}

\author{
Wei XIE, Brian BURKE \\ Laboratory of Nuclear Dynamics and Architecture, Skin Research Institute of Singapore (SRIS), Agency for Science, Technology and Research (A*STAR), \\ Singapore. \\ ${ }^{*}$ Correspondence: brian.burke@sris.a-star.edu.sg \\ https://doi.org/10.37175/stemedicine.v2i5.73
}

\begin{abstract}
In metazoans, a thin filamentous network referred to as the nuclear lamina plays an essential role in providing mechanical support to the nucleus. The major constituent of the nuclear lamina is type $\mathrm{V}$ intermediate filament proteins that are collectively referred to as lamins. A variety of diseases collectively termed laminopathies have been linked to mutations in genes encoding nuclear envelope proteins, in particular lamins, such as X-linked Emery Dreifus muscular dystrophy, dilated cardiomyopathy, Dunnigan type familial partial lipodystrophy and Hutchinson-Gilford progeria syndrome. Apart from laminopathies, genome-wide association studies have also been implicated nuclear lamins in the pathophysiology of type 2 diabetes mellitus, although little information in terms of the function of lamins in its pathogenesis. Our current review attempts to summarize risk factors of diabetes mellitus that could be attributable to lamin mutations and indirectly linked to lamin-associated factors identified in the last two decades.
\end{abstract}

Keywords: Lamins · Diabetes mellitus · Nuclear lamina · Laminopathy · Dunnigan type familial partial lipodystrophy

\section{The nucleus and nuclear lamins}

Eukaryotic cells are defined by the presence of a nucleus enclosed by a pair of lipid bilayers, the outer nuclear membrane (ONM) and the inner nuclear membrane (INM), that together comprise the nuclear envelope (NE) (1). In metazoans, a thin filamentous network lining the nuclear face of the INM, which is referred to as the nuclear lamina, plays an essential role in providing mechanical support both to the NE itself and to the nucleus as a whole. In addition, the lamina provides anchoring sites at the nuclear periphery for higher order chromatin domains. In the way, the nuclear lamina represents a key element in the maintenance of global nuclear architecture (2). The major constituent of the nuclear lamina are type $\mathrm{V}$ intermediate filament (IF) proteins that are collectively referred to as lamins. Like all IF proteins, lamins will spontaneously self-associate to form filaments. The lamin filament network displays multiple interactions with integral proteins of the INM as well as with

Received: Nov 26, 2020; Accepted: Dec 22, 2020.

(c) The Author(s). 2020 This is an Open Access article distributed under the terms of the Creative Commons License (http://creativecommons.org/licenses/by/4.0/) which permits unrestricted use, distribution, and reproduction in any medium or format, provided the original work is properly cited. chromatin proteins (3). In addition, the lamina shares associations with nuclear pore complexes. These massive macromolecular structures are situated at annular junctions between the INM and ONM and regulate the exchange of macromolecules between the nucleus and cytoplasm. Besides having a critical structural role in the overall architecture of the nucleus, the nuclear lamins are pivotal for other aspects of normal nuclear function. In addition to regulating chromatin, particularly heterochromatin distribution, the lamins have been implicated in aspects of DNA replication and DNA damage repair (4-7).

The family of lamin proteins fall into two broad classes based on sequences, A-type and B-type. In humans, the two major A-type lamins, $\mathrm{A}$ and $\mathrm{C}$, are encoded by the LMNA gene and are derived by alternative splicing of the same primary transcript. Two minor isoforms A $\Delta 10$ and testis-specific C2, are similarly encoded by LMNA. The major B-type lamins, B1 and B2, are encoded by the LMNB1 and LMNB2 genes, respectively. Lamin B3, a minor testis-specific B-type lamin isoform is an alternatively spliced product of LMNB2. A type lamins have roughly neutral isoelectric points and are found in the majority of adult cell types. However, they are absent from early embryonic cells as well as from some stem cell niches. In contrast, the $\mathrm{B}$ type lamins, which have acidic 

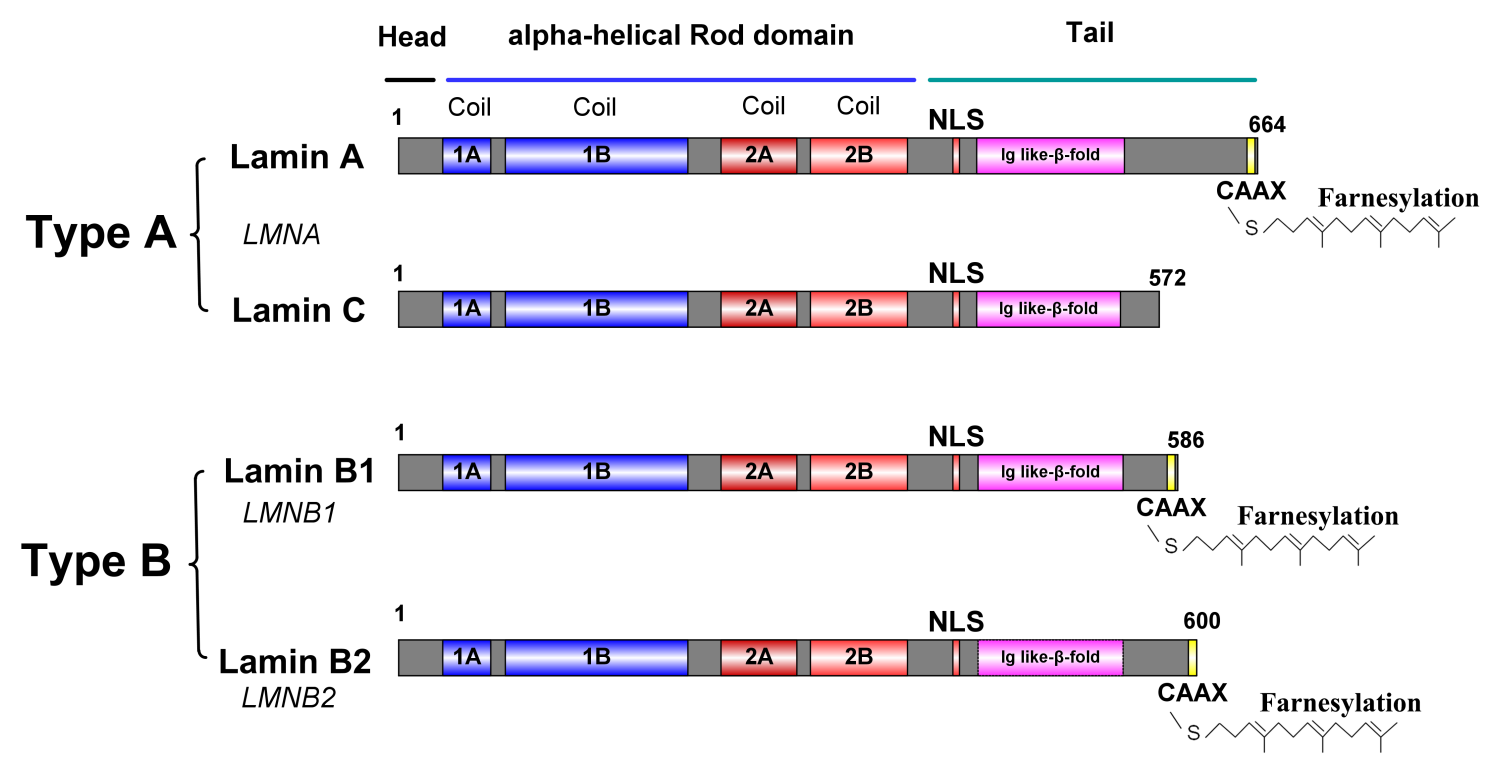

Figure 1. Schematic illustration of type A and B lamins. NLS, nuclear localization sequence.

isoelectric points, are, as a class, constitutively expressed, with lamin B1 and/or lamin B2 found in all nucleated cell types, both embryonic and adult (8).

All lamin proteins share the characteristic IF protein structure: a central $\alpha$-helical coiled-coil "rod domain", a non- $\alpha$-helical "head" at the N-terminus, and a globular "tail" at the C terminus (Figure 1). Unique to lamins, the core of this tail domain is organized as an immunoglobulin fold. Between the Ig-like $\beta$-fold domain and the central rod domain is a basic SV40-like nuclear localization sequence (NLS) that is essential for correct trafficking of newly synthesized lamins across the NE via NPCs. All mammalian lamins, with the exception lamin C, contain a C-terminal Ras-like CAAX motif that featuring a cysteine residue $(C)$ followed by two aliphatic amino acid residues (AA), and terminating with any other amino acid (X, but usually methionine or leucine). The CAAX motif represents a site of prenylation (farnesylation in the case of the lamins). This takes very soon after release of the newly synthesised protein from the ribosome and is catalyzed by a farnesyl transferase that attaches the 15-carbon farnesyl lipid group to the CAAX cysteine via a thioether bond. Farnesylation is followed immediately by proteolysis of the AAX tripeptide. In the case of the B-type lamins this is accomplished by Rce1 (Ras converting enzyme 1) whereas lamin A utilizes ZmpSte24, a membrane associated protease. Removal of the AAX tripeptide exposes the farnesylated cysteine as the new C-terminus. This is then carboxymethylated by isoprenylcysteine carboxymethyl transferase (ICMT). Carboxymethylation represents the completion of C-terminal processing of the B-type lamins. However, in the case of lamin A an additional proteolysis step mediated by ZmpSte24 removes the C-terminal 14 amino acids to generate the mature protein. This final proteolysis step occurs within about an hour of synthesis but after pre-lamin A has been incorporated into the nuclear lamina.
In this way, while B-type lamins remain permanently farnesylated, lamin A retains this modification only transiently. This divergence in C-terminal processing has marked consequences in terms of the behaviour of A-type versus B-type lamins. This is most notable during mitosis during which time the NE, including the lamina, is broken down so that the condensed chromosomes can engage with the mitotic spindle, a cytoplasmic structure in higher cells. While A-type lamins become dispersed as soluble proteins throughout the mitotic cytosol, the B-type lamins remain membrane-associated. As will be seen below, the effects of differential farnesylation on lamin function have important consequences in terms of our understanding of diseases associated with lamin defects $(9,10)$.

\section{Lamins and associated diseases}

A variety of diseases have now been linked to mutations in genes encoding NE proteins. The first of these to be described was X-linked Emery Dreifus muscular dystrophy (EDMD), which is caused by defects in an INM protein known as Emerin. EDMD is characterized by early contractures in the Achilles tendons and at the elbows, muscle wasting and cardiac conduction system defects associated with dilated cardiomyopathy. Later an autosomal dominant form of EDMD was mapped to mutations within LMNA. Studies employing a Lmna deficient mouse strain, which develops a syndrome that bears a striking resemblance to human EDMD, revealed that Lamin A and C can associate with Emerin and that these two lamins are required for Emerin localization to the INM, at least in fibroblasts and muscle cells. Multiple other diseases are also caused by mutations in LMNA. These include dilated cardiomyopathy (DCM), limb girdle muscular dystrophy, Dunnigan type familial partial lipodystrophy (FPLD), a peripheral neuropathy (Charcot Marie Tooth disease, CMT2) and Hutchinson-Gilford progeria syndrome (HGPS), a premature aging disorder. 
HGPS, an extremely rare disease, is most frequently caused a 50-residue deletion within the lamin A tail which eliminates the ZmpSte24 cleavage site. This results in the accumulation of a truncated form of lamin A which is permanently farnesylated. Loss of ZmpSte24 itself results in Restrictive Dermopathy, a rare recessive perinatal lethal disorder. An intriguing aspect to this is that lamin A is the only known substrate of this protease.

The range of diseases caused by LMNA mutations has been a matter of considerable scrutiny over the issues. An issue here is the fact that LMNA is expressed in the vast majority of adult cell types. However, different LMNA mutations affect different tissues, ranging from striated muscle to adipose and neuronal tissues. In addition, metabolic perturbations may also be observed to a greater or lesser extent. One explanation for this curious situation is that lamin defects may compromise the structural integrity of the cell nucleus leading to DNA damage and cell death. This is very likely the case in mechanically stressed tissues such as striated muscle. However, it cannot account for diseases such lipodystrophy. FPLD in particular is caused by mutations in the Lamin $\mathrm{A} / \mathrm{C}$ tail domain in the vicinity of Arginine 482. These mutations have seemingly little or no effect on striated muscle. Consequently, it has been suggested that FPLD is caused by lamin-dependent transcriptional perturbations. An attractive possibility is that certain lamin mutations affect interactions with other components that are themselves expressed in a tissue-specific manner. It is most likely that all of these effects contribute to LMNA linked diseases with their differential impacts varying according to tissue type.

The majority of disease-causing mutations in genes encoding NE proteins are found within LMNA. As alluded to above several hundred such deleterious LMNA mutations have been identified. Other disorders, including $\mathrm{X}$-linked EDMD and adult onset autosomal dominant leukodystrophy occur as a result of mutations in EMD, the gene encoding emerin, and LMNB1 respectively, the latter involving a gene duplication. Similarly, mutations in LEMD3, encoding an INM protein, MAN1, are associated with Buschke-Ollendorff syndrome. Mutations in LBR, encoding another INM protein, lamin B receptor, variously cause Pelger-Huet anomaly and Greenberg Skeletal Dysplasia. These major lamin-associated disorders are collectively termed "laminopathies" and have been described in detail in an excellent review by Worman (11). Our current discussion focuses on a much more widely spread disease particularly in developed countries - diabetes mellitus (DM) and the possible role of A-type lamins.

\section{Diabetes mellitus (DM)}

DM typically involves inadequate insulin activity and/ or secretion, which often leads to chronic hyperglycemia and impaired metabolism of lipids, proteins, and carbohydrates (12). There has been a continuing rise of DM in developed countries, including both Singapore and the United States. Two major types of diabetes have been described, namely insulin-dependent diabetes mellitus (type 1 DM, T1DM) as well as non-insulin-dependent diabetes mellitus (type 2 DM, T2DM), the latter of which is the more common, accounting for $90 \%-95 \%$ of all DM cases (13). Furthermore, incidences of T2DM has been estimated to increase to approximately 439 million by 2030 (14), thereby posing a serious global public health problem. It has been widely established that T1DM and T2DM are characterized by the aberrant functions or even failures of pancreatic $\beta$-cells. Hyperglycemia in T2DM is caused by the gradual aggravation of insulin resistance at peripheral sites such as in the adipose or muscle tissues, in addition to pancreatic $\beta$-cell dysfunction. Hence, it is of great value to investigate the intracellular events that take place within the pancreatic $\beta$-cells. Understanding of such events will facilitate the identification of potential drug targets for managing and/or preventing DM.

Although the pathophysiology of T2DM has not been fully elucidated, genetic risk factors are widely considered to be a major component of the disease. Genomewide association studies (GWAS), which analyze the correlations between common diseases and singlenucleotide polymorphisms (SNPs) through comparisons of the genetic features of patients and healthy subjects, have identified a number of susceptibility loci of T2DM (15). Some of these polymorphisms map to chromosome location 1q21.2 that harbours the LMNA gene.

Apart from the GWAS, there has been little information in terms of the function of lamins in the pathogenesis of DM. This review attempts to summarize risk factors of T2DM that could be attributable to lamin mutations and indirectly linked to lamin-associated factors identified in the last two decades.

\section{LMNA mutations associated with DM \\ Dunnigan-type familial partial lipodystrophy (FPLD)}

Mutations in LMNA gene were first identified to be the cause of Dunnigan-type familial partial lipodystrophy (FPLD) through an inspired piece of intuitive detective work (16). FPLD patients typically present symptoms that include subcutaneous fat loss, glucose intolerance, hypertension and insulin resistance, along with other characteristic changes associated with metabolic syndrome that eventually lead to DM (Figure 2). LMNA was one of more than 100 positional candidates implicated in FPLD. However, Bonne and colleagues realised that the sitespecific pattern of fat loss in FPLD was quite similar to the pattern of muscle wasting in EDMD, which had only recently been linked to LMNA mutations (17). Sequence analyses of the LMNA locus in FPLD patients revealed that mutations in exon 8, typically in the codon for R482 in the Ig-fold region of the tail domain (and in rare cases, codons $465,486,582$ or 584) were responsible for FPLD (18-21).

Since the original linkage of FPLD to LMNA mutations within the Ig fold, there has been speculation that these might adversely affect the stability of lamin A and C multimers, thereby compromising the integrity of the nuclear lamina as a whole. This could then impact adipocyte function as a result of abnormal interactions between lamins and transcription factors, nuclear 


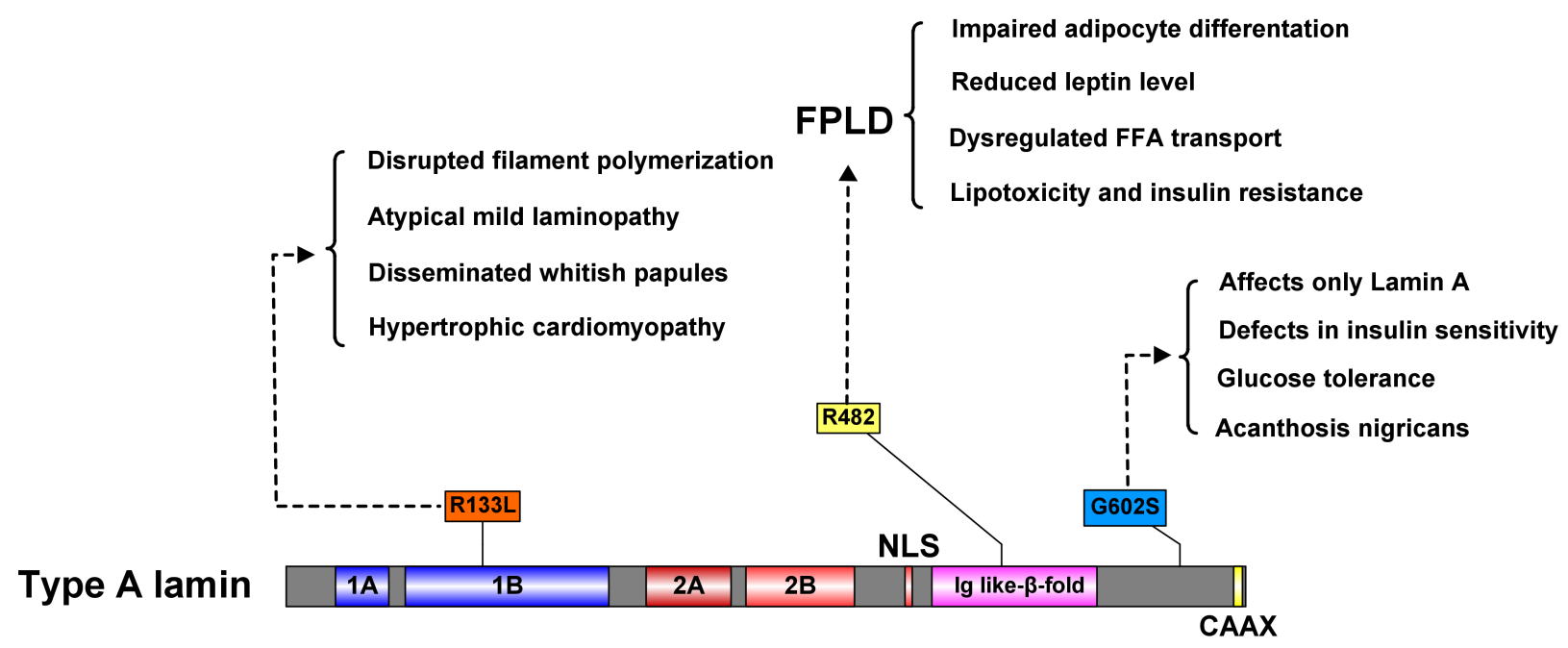

Figure 2. LMNA mutations associated with diabetes mellitus (DM). R482 mutation leads to Dunnigan-type familial partial lipodystrophy (FPLD), showing symptoms such as subcutaneous fat loss, glucose intolerance, hypertension, and insulin resistance that eventually lead to DM. R133L mutation leads to atypical mild laminopathy, showing lipodystrophy and insulin resistance that are characteristic of type A insulin resistance syndrome. G602S mutation affects only lamin A protein and leads to insulin sensitivity, glucose tolerance and acanthosis nigricans, and is significantly associated with T2DM risk.

membrane proteins, chromatin, and/or other cellular proteins (22). The mutant LMNA has also been suggested to impair adipocyte differentiation, preadipocyte proliferation and modulation of apoptosis, all of which could lead to decreased adipose tissue mass (23). On the other hand, the plasma level of leptin was significantly lower in FPLD individuals with the LMNA R482 mutations (24). Leptin is a peptide hormone that depresses hunger and is therefore an important metabolic regulator. Deficiency of leptin is associated with insulin resistance (25). As peripheral adipocytes are the primary producer of circulating leptin, it is reasonable to conclude that decreased adipose tissue mass (a consequence of the LMNA mutation) may lead to lowered leptin production or secretion. From a functional perspective, leptin aids the preferential delivery of free fatty acids (FFA) to adipocytes, versus liver and skeletal muscle (26). Therefore the leptin deficiency in FPLD may augment the possible FFA exposure to non-adipocytes, causing lipotoxicity and insulin resistance $(27,28)$.

\section{R133L LMNA mutation leading to atypical mild laminopathy}

FPLD was initially regarded as the only form of laminopathy-related DM. However, subsequent investigations demonstrated that DM and insulin resistance could be an important hallmark of more complex laminopathy phenotypes. Caux et al. described a case study of an atypical milder form of laminopathy with features that overlapped with FPLD (29). The patient was initially diagnosed with hepatic steatosis, hypertriglyceridemia and insulin-resistant DM. As the patient also suffered lipodystrophy as well as insulin resistance that was characteristic of type A insulin resistance syndrome, usually caused by mutations of the insulin gene, molecular assays on insulin receptors and seipin were first conducted, only to find no defect in any of these genes.

A later screen for mutations in the LMNA gene revealed that a heterozygous R133L LMNA mutation was responsible for the symptoms (Figure 2). Other symptoms typically associated with LMNA gene mutations were later identified, including disseminated whitish papules and hypertrophic cardiomyopathy with valvular involvement. Arginine 133 locates in a charged peptide segment that is highly conserved in vertebrate lamin $\mathrm{A} / \mathrm{C}$. The change from arginine (positively charged) to leucine (hydrophobic) within the lamin $\mathrm{A} / \mathrm{C}$ coiled-coil dimerization domain would potentially compromise the polymerization of lamins and subsequent assembly of lamin filaments. Consistent with this speculation, nuclear abnormalities were indeed reported in cultures of primary fibroblasts from this patient.

\section{G602S LMNA mutation}

In another case study of a non-obese patient without lipodystrophy, the type A insulin resistance syndrome was linked to a newly-identified heterozygous G602S mutation in LMNA gene (30). Different from the earlier mentioned R133L mutation that impairs both lamins A and C, G602 resides in exon 11 therefore this missense mutation affects only the lamin A protein. Nuclear shape in the patient fibroblasts also appeared abnormal, not dissimilar to that observed in the patient with R133L mutation. Most significantly, this G602S LMNA mutation is accompanied by defects in insulin sensitivity, glucose tolerance, and acanthosis nigricans, therefore indicating that LMNA mutations can impair the insulin signalling, even without signs of clinical lipodystrophy.

In addition to this case study, a stronger link between G602S mutation and T2DM was recently established in a large scale, ethnically diverse population study involving 
over 60,000 unrelated individuals in the ExAC cohort $(31,32)$. LMNA variants were independently analysed via the T2D Knowledge Portal, and the results revealed that G602S was significantly associated with T2DM and could serve as a risk factor for DM among African Americans.

\section{Lamin A/C expression and T2DM}

Besides lamin A, the other product of the LMNA gene, lamin $\mathrm{C}$ has also been implicated in the pathological processes of DM. Using a mouse model that expresses exclusively lamin $\mathrm{C}$, Toledo et al. reported that when challenged with DM-inducing conditions, these mice were able to normalize their fasting glycemia through enhancing both the regeneration of $\beta$-cells and the secretion of insulin, while the mice expressing both lamins A and $\mathrm{C}$ exhibited disrupted glucose homeostasis (33). The capacity to regenerate $\beta$-cells in the lamin $\mathrm{C}$ only expressing/lamin A deficient mice suggested an alternative therapeutic potential for DM treatment through regulating LMNA splicing, because an insufficient supply of functional insulin-producing $\beta$-cells is a clinical feature commonly found in T1DM and T2DM.

There is, however, one caveat in the above study: it remains unclear that whether the lack of lamin $A$ expression indeed causes the observed $\beta$-cell regeneration phenotype or the sole lamin $\mathrm{C}$ expression (or an imbalance of the two) is actually responsible. This possibility is certainly echoed by three independently earlier reports that, in adipose tissue samples collected from obese subjects and T2DM patients, expression of lamin $\mathrm{A} / \mathrm{C}$ is upregulated (34-36).

Later on, Kim et al. have brought the discovery one step forward, and found that the upregulated lamin A/C mainly occurs in adipose tissue macrophages (ATMs), and in particular, the epididymal white adipose tissue (eWAT), where it triggers enhanced production of pro-inflammatory cytokines through activating NF- $\kappa \mathrm{B}$ (37). Given the fact that eWAT contains more inflammatory cells and higher expression levels of proinflammatory genes (38-41), it can be speculated that in the ATM the elevated lamin $\mathrm{A} / \mathrm{C}$ increases expression levels of pro-inflammatory cytokines and that it is these that are mechanistically linked to insulin resistance (41-44). This hypothesis is certainly supported by observations in the same study, that deficiency in myeloid cell-specific lamin A/C attenuated obesity-associated inflammation in adipose tissues and resistance to insulin (37). These findings collectively indicate that in ATMs, lamin A/C acts as a novel regulator in obesity-elicited insulin resistance and inflammatory responses in adipose tissues.

\section{Lamin-associated factors linked to DM}

Apart from being implicated in the upstream events that affect insulin production/resistance, nuclear lamins are also implicated in the transcriptional modification of downstream insulin-response machinery through its associated factors (Figure 3).

An exemplary case, illustrated by the work of Pak and colleagues, is Caveolin-2 $(45,46)$. Caveolins are a group of lipid raft-associated integral membrane proteins that participate in diverse cell signal transduction and mechanotransduction processes (47). It has been reported that upon insulin stimulation, Caveolin-2 is transported to the INM, where it initiates direct interaction with Type A lamins (45) and subsequently undergoes phosphorylation at Tyr-19 and mono-oligomerization (48). Interestingly, the maintenance of pY19-Caveolin-2 oligomer is dependent on A-type lamins (46). By interacting with lamin A or C, pY19-Caveolin-2 decreases histone H3 lysine 9 trimethylation ( $\mathrm{H} 3 \mathrm{~K} 9 \mathrm{me} 3$ ), thereby opening the lamin $\mathrm{A} / \mathrm{C}$ sequestered (transcription-inactivated) chromatin domains containing the promoters of insulin-response genes such as Elk-1 and JunB, as well as signal transducer and activator of transcription 3 (STAT3) $(49,50)$. Besides releasing transcriptionally repressed chromatin domains, the lamin A/C associated pY19-Caveolin-2 also actively promotes transcription of the insulin-response genes by recruiting RNA polymerase II to promoter regions through increased acetylations at histone $\mathrm{H} 3$ lysines 9, 18 and 27 (45). In this way, Caveolin-2 acts as an A-type lamin dependent epigenetic activator of insulin-response genes.

In addition to modulating transcriptional activity through histone modification, lamin $\mathrm{A} / \mathrm{C}$ is also able to regulate the retinoblastoma protein $(\mathrm{RB})$ and $\mathrm{E} 2$-factor $(\mathrm{E} 2 \mathrm{~F})$ family of transcription factors (51). The regulation of $\mathrm{RB}$, a nuclear lamina-associated transcription factor, is carried out by lamina-associated-polypeptide- $2 \alpha$ (LAP $2 \alpha$ ) and A-type lamins, where lamin $\mathrm{A} / \mathrm{C}$ acts to promote subnuclear localization of RB, as well as to stabilize $\mathrm{RB}$ against proteasome-mediated degradation (52). In pancreas-selective conditional knockout mice, lamin $\mathrm{A} / \mathrm{C}$ deficiency destabilizes RB to activate E2F, leading to ER stress, loss of acinar cell markers, enhanced autophagy, proliferation, as well as apoptosis, eventually leading to chronic pancreatitis (51). As the RB-E2F pathway is critical for lipogenesis in liver, pancreas, and adipocytes (53-55), the regulation of RB stability by A-type lamins proposed by Elenbaas et al. is also of particular relevance to FPLD. Taken together, all of these reports imply that A-type lamins have a hitherto under-appreciated role in the regulation of energy metabolism in diverse cell types.

\section{Perspective}

T2DM, as well as its complications, has posed as an increasingly severe health issue worldwide especially in developed economies. Studying the underlying causes, in particular genetic factors, is crucial in the prevention and treatment against T2DM. In the current review, we have discussed one of such risk factors of diabetes mellitus: the nuclear lamins. In addition to laminopathies, which are mainly caused by mutations in lamins and associated proteins, lamin mutations also contributes, directly or indirectly, to diabetic symptoms. Understanding the correlation between lamin mutations with T2DM could make the clinical management of patients affected by both more effective. 


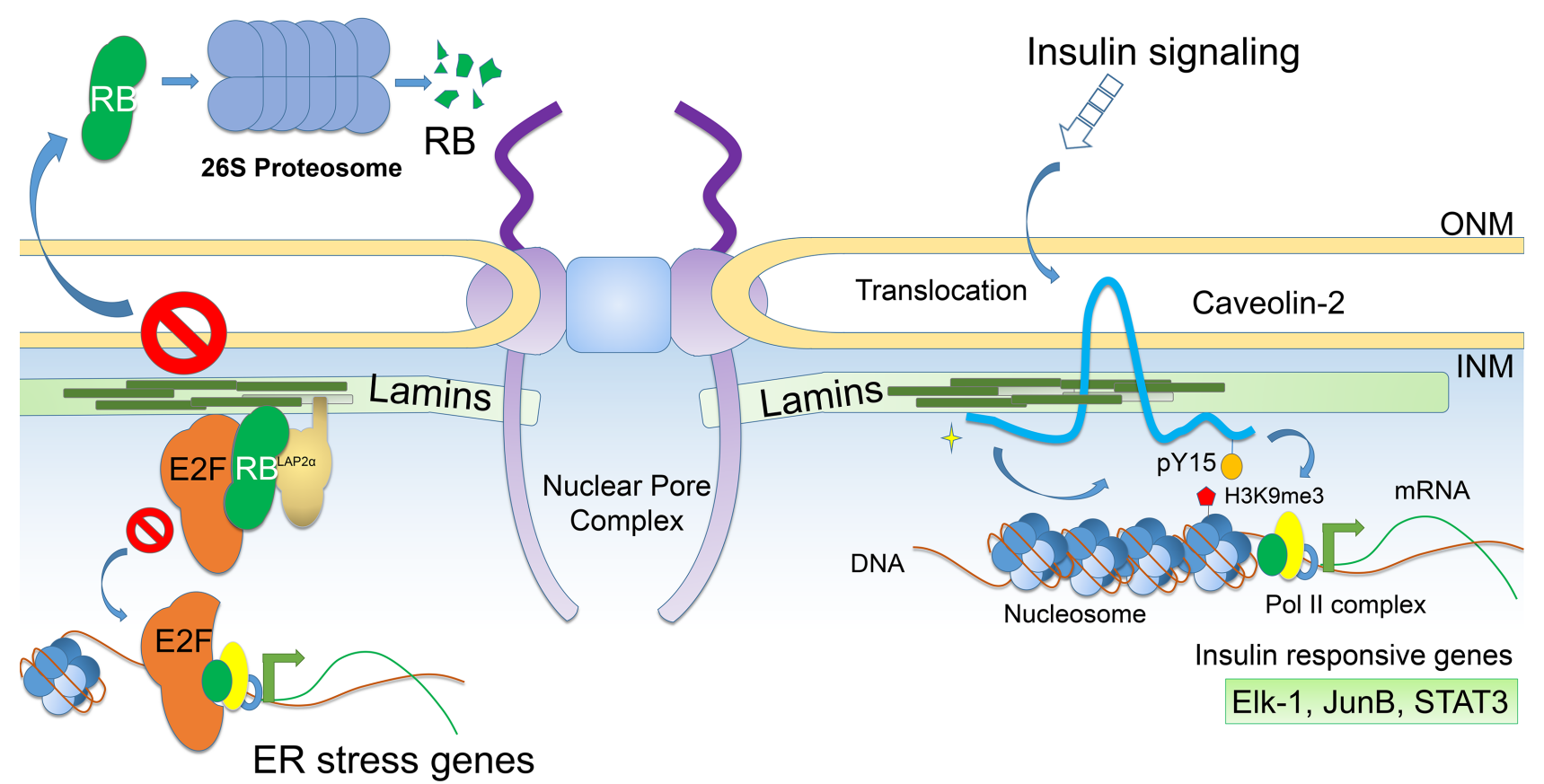

Figure 3. Lamin-associated factors linked to diabetes mellitus (DM). Apart from being implicated in the upstream events that affects insulin production/resistance, nuclear lamins are also suggested in the transcriptional modification of downstream insulin-response and ER stress response machinery through its associated factors.

\section{Acknowledgement}

This work is supported by the Agency for Science, Technology and Research $\left(A^{*} S T A R\right)$ of Singapore.

\section{Conflict of interest}

The authors declare that there is no conflict of interests regarding the publication of this paper.

\section{References}

1. Romero-Bueno R, de la Cruz Ruiz P, Artal-Sanz M, Askjaer $P$, Dobrzynska A. Nuclear organization in stress and aging. Cells. 2019;8(7).

2. Xie W, Burke B. Nuclear networking. Nucleus. 2017;8(4):323-30.

3. Polychronidou M, Grobhans J. Determining nuclear shape: the role of farnesylated nuclear membrane proteins. Nucleus. 2011;2(1):17-23.

4. Harr JC, Luperchio TR, Wong X, Cohen E, Wheelan SJ, Reddy KL. Directed targeting of chromatin to the nuclear lamina is mediated by chromatin state and A-type lamins. J Cell Biol. 2015;208(1):33-52.

5. Butin-Israeli V, Adam SA, Jain N, Otte GL, Neems D, Wiesmuller L, et al. Role of lamin b1 in chromatin instability. Mol Cell Biol. 2015;35(5):884-98.

6. Butin-Israeli V, Adam SA, Goldman RD. Regulation of nucleotide excision repair by nuclear lamin b1. PLoS One. 2013;8(7):e69169.

7. Earle AJ, Kirby TJ, Fedorchak GR, Isermann P, Patel J, Iruvanti $S$, et al. Mutant lamins cause nuclear envelope rupture and DNA damage in skeletal muscle cells. Nat Mater. 2020;19(4):464-73.

8. Xie W, Burke B. Lamins. Curr Biol. 2016;26(9):R348-50.

9. Burke $B$, Stewart $C L$. The nuclear lamins: flexibility in function. Nat Rev Mol Cell Biol. 2013;14(1):13-24.
10. Dechat T, Adam SA, Taimen P, Shimi T, Goldman RD. Nuclear lamins. Cold Spring Harb Perspect Biol. 2010;2(11):a000547.

11. Worman HJ. Nuclear lamins and laminopathies. J Pathol. 2012;226(2):316-25

12. Wu Y, Ding Y, Tanaka $Y$, Zhang W. Risk factors contributing to type 2 diabetes and recent advances in the treatment and prevention. Int J Med Sci. 2014;11(11):1185-200.

13. Tripathi BK, Srivastava AK. Diabetes mellitus: complications and therapeutics. Med Sci Monit. 2006;12(7):RA130-47.

14. Chen L, Magliano DJ, Zimmet PZ. The worldwide epidemiology of type 2 diabetes mellitus--present and future perspectives. Nat Rev Endocrinol. 2011;8(4):228-36.

15. Thomas PPM, Alshehri SM, van Kranen HJ, Ambrosino E. The impact of personalized medicine of Type 2 diabetes mellitus in the global health context. Per Med. 2016;13(4):381-93.

16. Hegele RA. Familial partial lipodystrophy: a monogenic form of the insulin resistance syndrome. Mol Genet Metab. 2000;71(4):539-44.

17. Bonne G, Mercuri E, Muchir A, Urtizberea A, Becane HM, Recan D, et al. Clinical and molecular genetic spectrum of autosomal dominant Emery-Dreifuss muscular dystrophy due to mutations of the lamin A/C gene. Ann Neurol. 2000;48(2):170-80.

18. Cao H, Hegele RA. Nuclear lamin A/C R482Q mutation in canadian kindreds with Dunnigan-type familial partial lipodystrophy. Hum Mol Genet. 2000;9(1):109-12.

19. Garg A, Vinaitheerthan M, Weatherall PT, Bowcock AM. Phenotypic heterogeneity in patients with familial partial lipodystrophy (dunnigan variety) related to the site of missense mutations in lamin a/c gene. J Clin Endocrinol Metab. 2001;86(1):59-65.

20. Vigouroux C, Magre J, Vantyghem MC, Bourut C, Lascols $\mathrm{O}$, Shackleton $\mathrm{S}$, et al. Lamin A/C gene: sex-determined expression of mutations in Dunnigan-type familial partiallipodystrophy and absence of coding mutations in 
congenital and acquired generalized lipoatrophy. Diabetes. 2000;49(11):1958-62.

21. Hegele RA, Cao H, Anderson CM, Hramiak IM Heterogeneity of nuclear lamin A mutations in Dunnigantype familial partial lipodystrophy. J Clin Endocrinol Metab. 2000;85(9):3431-5.

22. Morris GE, Manilal S. Heart to heart: from nuclear proteins to Emery-Dreifuss muscular dystrophy. Hum Mol Genet. 1999;8(10):1847-51.

23. Flier JS. Pushing the envelope on lipodystrophy. Nat Genet. 2000;24(2):103-4.

24. Hegele RA, Cao H, Huff MW, Anderson CM. LMNA R482Q mutation in partial lipodystrophy associated with reduced plasma leptin concentration. J Clin Endocrinol Metab. 2000;85(9):3089-93.

25. Petersen MC, Shulman GI. Mechanisms of insulin action and insulin resistance. Physiol Rev. 2018;98(4):2133-223.

26. Unger RH, Zhou YT, Orci L. Regulation of fatty acid homeostasis in cells: novel role of leptin. Proc Natl Acad Sci U S A. 1999;96(5):2327-32

27. Randle PJ. Regulatory interactions between lipids and carbohydrates: the glucose fatty acid cycle after 35 years. Diabetes Metab Rev. 1998;14(4):263-83.

28. Shulman Gl. Cellular mechanisms of insulin resistance in humans. Am J Cardiol. 1999;84(1A):3J-10J.

29. Caux F, Dubosclard E, Lascols O, Buendia B, Chazouilleres $\mathrm{O}$, Cohen $\mathrm{A}$, et al. A new clinical condition linked to a novel mutation in lamins $A$ and $C$ with generalized lipoatrophy, insulin-resistant diabetes, disseminated leukomelanodermic papules, liver steatosis, and cardiomyopathy. J Clin Endocrinol Metab. 2003;88(3):1006-13.

30. Young J, Morbois-Trabut L, Couzinet B, Lascols O, Dion $E$, Bereziat $V$, et al. Type $A$ insulin resistance syndrome revealing a novel lamin A mutation. Diabetes. 2005;54(6):1873-8.

31. Lek M, Karczewski KJ, Minikel EV, Samocha KE, Banks E, Fennell T, et al. Analysis of protein-coding genetic variation in 60,706 humans. Nature. 2016;536(7616):285-91

32. Florwick A, Dharmaraj T, Jurgens J, Valle D, Wilson KL. LMNA sequences of 60,706 unrelated individuals reveal 132 novel missense variants in A-type lamins and suggest a link between variant p.G602S and type 2 diabetes. Front Genet. 2017;8:79

33. de Toledo M, Lopez-Mejia IC, Cavelier P, Pratlong M, Barrachina C, Gromada X, et al. Lamin C counteracts glucose intolerance in aging, obesity, and diabetes through beta-cell adaptation. Diabetes. 2020;69(4):647-60.

34. Miranda M, Chacon MR, Gutierrez C, Vilarrasa N, Gomez $\mathrm{JM}$, Caubet $\mathrm{E}$, et al. LMNA mRNA expression is altered in human obesity and type 2 diabetes. Obesity (Silver Spring). 2008;16(8):1742-8.

35. Rodriguez-Acebes S, Palacios N, Botella-Carretero JI, Olea N, Crespo L, Peromingo R, et al. Gene expression profiling of subcutaneous adipose tissue in morbid obesity using a focused microarray: distinct expression of cell-cycleand differentiation-related genes. BMC Med Genomics. 2010;3:61

36. Nadeau M, Noel S, Laberge PY, Hurtubise J, Tchernof A. Adipose tissue lamin $A / C$ messenger RNA expression in women. Metabolism. 2010;59(8):1106-14.

37. Kim Y, Bayona PW, Kim M, Chang J, Hong S, Park Y, et al. Macrophage lamin $A / C$ regulates inflammation and the development of obesity-induced insulin resistance. Front Immunol. 2018;9:696.

38. Fox CS, Massaro JM, Hoffmann U, Pou KM, MaurovichHorvat $\mathrm{P}$, Liu CY, et al. Abdominal visceral and subcutaneous adipose tissue compartments: association with metabolic risk factors in the Framingham Heart Study. Circulation. 2007;116(1):39-48.
39. Ledoux S, Queguiner I, Msika S, Calderari S, Rufat P, Gasc JM, et al. Angiogenesis associated with visceral and subcutaneous adipose tissue in severe human obesity. Diabetes. 2008;57(12):3247-57.

40. Ibrahim MM. Subcutaneous and visceral adipose tissue: structural and functional differences. Obes Rev. 2010;11(1):11-8.

41. Lee BC, Kim MS, Pae M, Yamamoto Y, Eberle D, Shimada $\mathrm{T}$, et al. Adipose natural killer cells regulate adipose tissue macrophages to promote insulin resistance in obesity. Cell Metab. 2016;23(4):685-98.

42. Lumeng CN, DelProposto JB, Westcott DJ, Saltiel AR. Phenotypic switching of adipose tissue macrophages with obesity is generated by spatiotemporal differences in macrophage subtypes. Diabetes. 2008;57(12):3239-46.

43. Li P, Lu M, Nguyen MT, Bae EJ, Chapman J, Feng D, et al. Functional heterogeneity of CD11c-positive adipose tissue macrophages in diet-induced obese mice. J Biol Chem. 2010;285(20):15333-45.

44. Kratz M, Coats BR, Hisert KB, Hagman D, Mutskov V, Peris $\mathrm{E}$, et al. Metabolic dysfunction drives a mechanistically distinct proinflammatory phenotype in adipose tissue macrophages. Cell Metab. 2014;20(4):614-25.

45. Jeong K, Kwon H, Lee J, Jang D, Pak Y. Insulin-response epigenetic activation of Egr-1 and JunB genes at the nuclear periphery by A-type lamin-associated pY19-Caveolin-2 in the inner nuclear membrane. Nucleic Acids Res. 2015;43(6):3114-27.

46. Kwon H, Lee J, Jeong K, Jang D, Choi M, Pak Y. A-type lamin-dependent homo-oligomerization for pY19-Caveolin-2 to function as an insulin-response epigenetic regulator. Biochim Biophys Acta. 2016;1863(11):2681-9.

47. Parton RG, del Pozo MA. Caveolae as plasma membrane sensors, protectors and organizers. Nat Rev Mol Cell Biol. 2013;14(2):98-112.

48. Kwon H, Jeong K, Hwang EM, Park JY, Pak Y. A novel domain of caveolin-2 that controls nuclear targeting: regulation of insulin-specific ERK activation and nuclear translocation by caveolin-2. J Cell Mol Med. 2011;15(4):888-908.

49. Kwon H, Jeong K, Hwang EM, Park JY, Hong SG, Choi WS, et al. Caveolin-2 regulation of STAT3 transcriptional activation in response to insulin. Biochim Biophys Acta. 2009;1793(7):1325-33.

50. Jeong $\mathrm{K}$, Kwon H, Lee J, Jang D, Hwang EM, Park JY, et al. Rab6-mediated retrograde transport regulates inner nuclear membrane targeting of caveolin-2 in response to insulin. Traffic. 2012;13(9):1218-33.

51. Elenbaas JS, Bragazzi Cunha J, Azuero-Dajud R, Nelson B, Oral EA, Williams JA, et al. Lamin A/C maintains exocrine pancreas homeostasis by regulating stability of RB and activity of E2F. Gastroenterology. 2018;154(6):1625-9 e8.

52. Johnson BR, Nitta RT, Frock RL, Mounkes L, Barbie DA, Stewart $C L$, et al. A-type lamins regulate retinoblastoma protein function by promoting subnuclear localization and preventing proteasomal degradation. Proc Natl Acad Sci U S A. 2004;101(26):9677-82.

53. Iglesias A, Murga M, Laresgoiti U, Skoudy A, Bernales I, Fullaondo $A$, et al. Diabetes and exocrine pancreatic insufficiency in E2F1/E2F2 double-mutant mice. J Clin Invest. 2004;113(10):1398-407.

54. Hansen JB, Jorgensen C, Petersen RK, Hallenborg P, De Matteis R, Boye HA, et al. Retinoblastoma protein functions as a molecular switch determining white versus brown adipocyte differentiation. Proc Natl Acad Sci U S A. 2004;101(12):4112-7.

55. Denechaud PD, Lopez-Mejia IC, Giralt A, Lai Q, Blanchet E, Delacuisine B, et al. E2F1 mediates sustained lipogenesis and contributes to hepatic steatosis. J Clin Invest. 2016;126(1):137-50. 